54 Cervista HPV+ cases, ISH HPV was positive in 52 cases. Three cases were tested negative by both Cervista HPV and ISH HPV testing assay. There were 5 cases with an equivocal result by ISH HPV testing assay but a negative Cervista HPV testing result. Overall, the concordance between the two assays was $88.7 \%(55 / 62)$.

Conclusions: The Cervista HPV assay shows a high concordance with the ISH HPV assay. Consequently, Cervista HPV assays can be used as a valid HPV testing assay in FFPE tissue of oropharyngeal squamous carcinomas.

\section{ADULT WILMS' TUMOUR: A CASE REPORT WITH REVIEW OF LITERATURE}

$\underline{\text { Rapinder Gupta }}$

St. Paul Hospital Bangalore, India

Background: Wilms' tumor is the commonest primary malignant renal tumor in childhood. Rarely, it may present in the adult age group.

Case presentation: We report a 48-year-old male presenting with flank pain and haematuria. Abdominal ultrasound revealed a right renal mass measuring $11 \times 10 \mathrm{~cm}$, and a clinical diagnosis of renal cell carcinoma was made. Nephrectomy was performed, and a final diagnosis of adult Wilms' tumor was made based on the criteria proposed by Kilton et al.

Conclusion: The possibility of an adult Wilms' tumor should be considered when a patient presents with pain in the flank and a renal mass. Rarity of the tumor favors documentation in literature.

\section{CLINICOPATHOLOGIC SIGNIFICANCE OF MIR-221/ 222 EXPRESSION IN BREAST CANCER}

$\underline{\text { Jae Moon Gwak }}^{1}$, Hyun Jeong Kim ${ }^{1}$, Eun Joo Kim ${ }^{1}$, So Yeon Park ${ }^{1,2}$

${ }^{1}$ Department of Pathology, Seoul National University Bundang Hospital, Seongnam, Gyeonggi, and ${ }^{2}$ Department of Pathology, Seoul National University College of Medicine, Seoul, Republic of Korea

Backgrounds: MicroRNAs (miRNAs) are known to play an important role in the tumorigenesis of breast cancer. Especially, miR-221/222 has been reported to be associated with promotion of epithelial-mesenchymal transition (EMT), down-regulation of estrogen receptor (ER)-alpha and tamoxifen resistance.

Aims: We aimed to study expression levels of miR-221/222 in human breast cancer samples and to analyze their relationship with clinicopathologic features of the tumor including hormone receptor status, breast cancer subtype, expression of EMT markers and clinical outcome of the patients.

Methods: Quantitative RT-PCR was performed to detect expression levels of the miR-221/222 in 129 invasive breast cancers. EMT marker expression [expression of vimentin, smooth muscle actin (SMA), osteonectin and N-cadherin; loss of E-cadherin] was evaluated by immunohistochemistry.

Results: miR-222 expression level was significantly higher in tumors with high histologic grade and high $\mathrm{Ki}-67$ proliferation index. High expression of miR-222 was associated with ERpositivity and HER2 amplification. It expression was significantly higher in luminal B and HER2-enriched subtypes than in luminal A subtype. However, miR-222 expression level was lower in tumors showing EMT marker expression (vimentin, SMA and osteonectin). Expression of miR-221 was not associated with any clinicopathologic features of breast cancer and EMT marker expression. In survival analysis, expression level of miR-221/222 was not associated with patients' prognosis.

Conclusions: The association of miR-221/222 expression with EMT is not evident in this study. Although miR-222 expression was increased in ER-positive breast cancers, its expression level was much higher in luminal B subtype than in luminal A subtype, suggesting that miR-222 may be related to aggressiveness and endocrine therapy resistance in ER-positive breast cancer.

\section{THE CLINICOPATHOLOGICAL FEATURES OF EXTRANODAL NK/T-CELL LYMPHOMA NASAL TYPE (NKTCL) IN UNIVERSITAS INDONESIA/CIPTO MANGUNKUSUMO HOSPITAL JAKARTA}

Agnes S. Harahap, Stephanie Marisca, Maria F. Ham, Endang S.R. Hardjolukito

Department of Anatomical Pathology, Faculty of Medicine Universitas Indonesia/Cipto Mangunkusumo Hospital, Jakarta, Indonesia

Background: Extranodal NK/T-cell lymphoma nasal type (NKTCL) is more common in Asia than in the Western countries. Classic morphological features of NKTCL include necrosis, angiocentricity and polymorphism. In small biopsy sample, these features are not always found, meanwhile there is no data regarding percentage of these features.

Aims: To identify the demographic profiles of our NKTCL cases, its morphological and immunohistochemistry (IHC) features and EBV positivity.

Methods: NKTCL cases during period of January 2010 to July 2014 were retrieved from our database. Data of age, sex, tumor location and initial diagnosis were recorded. Hematoxylin and eosin (HE), IHC, and EBER ISH slides were reviewed.

Results: Thirty seven cases were found increasing within years, showing male predominance (M:F=2:1) ranging from 18 to 67 years old with mean age of 43 . Nasal region $(75.7 \%)$ was the most affected sites, meanwhile skin and soft tissue (13.5\%), nasopharyngeal $(5.4 \%)$, intestinal $(5.4 \%)$ were the most common extranasal sites involved. Percentages of histopathological features found consecutively were: atypical cells (100\%), necrosis (91.9\%), angiocentricity $(70.3 \%)$, polymorphism $(64.9 \%)$, monomorphism (27\%), but none of granuloma ( $0 \%$ ) was found. Percentage of IHC positivity were $100 \%$ for granzyme B (35/35) and perforin (34/34), $97.3 \%$ (36/37) for CD3, 94.6\% (35/37) for CD56, 94.4\% (17/18) for CD16. EBER was positive in $83.3 \%$ (20/24) of cases.

Conclusion: Pathologists should always be cautious of NKTCL in the presence of atypical cells found in nasal biopsy regardless of the classic histopathological features. In our center, CD16 positivity was relatively high with lower EBER positivity.

\section{DISSEMINATED EPITHELIOID ANGIOSARCOMA PRESENTING AS MULTIPLE INTESTINAL POLYPS WITH ACTIVE BLEEDING}

Roumina Hasan $^{1}$, Vidya Monappa

${ }^{1}$ Melaka Manipal Medical College, and ${ }^{2}$ Kasturba Medical College, Manipal University, Manipal, India 